\title{
COVID-19 pandemic: aggressive research, vaccination, testing, and environmental sustainability are the way forward
}

\author{
Naveen Kumar Arora ${ }^{1} \cdot$ Piyush Pandey ${ }^{2} \cdot$ Dilfuza Egamberdieva $^{3} \cdot$ Tahmish Fatima $^{4}$
}

Published online: 9 September 2021

(c) Society for Environmental Sustainability 2021

Coronavirus disease 2019 (COVID-19) pandemic caused by severe acute respiratory syndrome coronavirus-2 (SARSCoV-2), first reported in December 2019, from Wuhan, China, has been declared as health, social and economic disaster unlike any other since World War II. The irreversible socio-economic changes brought by the pandemic have already disrupted the efforts to achieve the Sustainable Development Goals (SDGs) and even reversed the progress of some goals such as food security, inequality, health and education systems, economy, biodiversity, and environment conservation. Although some species of coronaviruses are well known as human pathogens, for example, severe acute respiratory syndrome coronavirus (SARS-CoV) and middle east respiratory syndrome coronavirus (MERS-CoV), both of which caused outbreaks in very recent past, yet mankind was caught by surprise and under-preparedness after the arrival of the contagion, COVID-19. It is important to research upon what went wrong because of which a novel virus ran through the globe and overwhelmed the health system in almost all the countries. Yes, there are successes seen in vaccine development and health systems, but have we learnt about the root cause of such pandemics related to frequent arrival of zoonotic pathogens in human population?

'Anthropocene epoch' with human population touching 8 billion, and anthropogenic activities causing huge impact on natural ecosystems have resulted in a raging environmental

Naveen Kumar Arora

nkarora.bbau@gmail.com

1 Department of Environmental Science, Babasaheb Bhimrao Ambedkar University, Lucknow, Uttar Pradesh, India

2 Department of Microbiology, Assam University, Silchar, Assam, India

3 Faculty of Biology, National University of Uzbekistan, Tashkent, Uzbekistan 100174

4 Department of Environmental Microbiology, Babasaheb Bhimrao Ambedkar University, Lucknow, Uttar Pradesh, India pandemic on the planet since last few decades. This has resulted in several issues including the transmission of novel zoonotic microbes to humans. As per World Health Organization (WHO) about $70 \%$ of the novel pathogens arriving in human population are zoonotic in nature. Research has shown that novel coronavirus is a zoonotic virus and most likely was transmitted to humans from wild animals. Bats and pangolins are the most common reservoirs of the coronaviruses. With a loss of around seven million hectares of dense forests each year; one million animal and plant species on the verge of extinction; world heading towards $3.2{ }^{\circ} \mathrm{C}$ rise in temperature by the end of this century; 75 per cent of the terrestrial and 66 per cent of marine areas being disturbed; the arrival of a zoonotic microbe in human population is becoming ever more likely (Mishra et al. 2021). The breaching of multilayered protection system of natural ecosystems due to deforestation, fragmentation of habitats, biodiversity loss resulting in extinction of natural hosts and climate change are the key factors driving the movement of zoonotic pathogens. Additionally, unplanned urbanization, easy transportation, poorly managed "wet markets" or slaughterhouses, trade in bush meat, warmer environment due to climate change and unorganized livestock cultivation are important factors as well. Global warming resulting in melting of permafrost is a warning sign for arrival of novel pathogens which are dormant since ages (Hofmeister et al. 2021). Apart from the environmental factors there are other challenges associated with a novel virus such as SARS-CoV-2. The development of mutants due to high rate of infection in the population is a challenge. Mutants can be more contagious, dangerous and may result in lower effectiveness of the vaccines. The pandemic is still in a dynamic phase and strategies must be developed accordingly to tackle the ever changing situation due to the virus. We need to be ahead of the evolving virus to control the pandemic as early as possible. In February 2021, International Science Council (ISC) has already established a multidisciplinary Oversight Panel with experts and technical team to report 
every 6-8 months about the possible scenarios developing due to COVID-19.

The positive aspect of COVID-19 pandemic has been the development of diverse vaccines based on different principles and platforms, in a very short response time. Some of these are novel vaccines. The mRNA technology is used for the first time to develop a vaccine. This may revolutionize the vaccine development in future for other deadly diseases and novel pathogens. The nimble plug-and-play concept along with faster development than conventional methods, mRNA technology can be the new dawn to curb future pandemics (Ball 2021). The vaccines approved by WHO (for COVID19) are using various technologies; Moderna-mRNA-1273, Pfizer/BioNTech-BNT162b2 (both m-RNA based), Janssen (Johnson \& Johnson)-Ad26.COV2.S (non-replicating viral vector), Oxford/AstraZeneca AZD1222 (non-replicating viral vector), Serum Institute of India-Covishield (Oxford/ AstraZeneca formulation; non-replicating viral vector), Sinopharm (Beijing)-BBIBP-CorV (Vero Cells; inactivated virus), Sinovac-CoronaVac (inactivated virus). There has not been any other pathogen/ disease for which such a wide variety of vaccines are available. National Institute of Health (NIH), United States, has already suggested the concept of booster dose to enhance the immunity against the variants of novel coronavirus. Also, National Institute of Allergy and Infectious Diseases (NIAID) collaborating with vaccine giant-Moderna has developed tweaked version of vaccine specifically for variants. Although a number of vaccines have been approved by WHO and several others rolled out by various countries, still there is inequality as far as availability (of vaccines) and vaccination of population is concerned in different parts of the globe. While in some of the low and middle income countries less than 5\% population is vaccinated, on the other hand there are countries which have already vaccinated more than half of their population. This inequality can lead to delay in controlling the pandemic and may also result in development of dangerous mutants which can even dodge the vaccines. Responding to the variants, epidemiologists and virologists propose the requirement of robust genomic and environmental surveillance system in order to halt the rampant spreading of viruses and detect the change in mechanism of transmission and even their influence on vaccination.

In last one year, molecular diagnosis of COVID-19 has also seen tremendous growth in technological upgradation and use. Till now, reverse transcription polymerase chain reaction (RT-PCR) based detection has been the most preferred and accepted diagnostic method, while serological-rapid antigen test, along with the use of metagenomic next generation sequencing (NGS) have aided testing and characterizing of SARS-CoV-2 RNA. The open platform Global Initiative on Sharing All Influenza Data (GISAID) already reports around 4,87,487 genome sequences of
SARS-CoV-2 virus across the globe (Joint WHO-China Study Team report 2021). Experts opine that without "deidentified meta-data" of patients from where the sequences were isolated, the modern genomic technology would be incapable of explaining the cause of mutation, the targeted population of the variant, or their mechanism of action. Furthermore, learning from the past, the present pandemic also requires analysis of various environmental matrices to inhibit their transmission pathways or use as an early warning system. WHO also approved the use of early warning systems for identifying the virus outbreak in a particular region. Studies have shown the presence of SARS-CoV-2 RNA in wastewater and that the virus may remain infectious for days to weeks in sewage (WHO 2020). Sewage water can thus be a good indicator to check the presence of the virus in a particular community. This can cut the cost and also help in taking proper measures to confiscate the virus. Detection of IgG antibodies in sewage can also be used as an early sign for the spiking cases. Renninger et al. (2021) demonstrated that surveillance of indoor dusts could be a potential matrix for the monitoring of disease transmission in high-risk populations and buildings. Additionally, other practical scenarios required to be emphatically considered are interior spaces, especially densely crowded with poor ventilation, air conditioning ducts; logistic chain, especially cold food-related environment due to the low-temperature resistance of $\mathrm{CoVs}$; and treatment plants along with the recipient water body (Yao et al. 2021). Several countries have established various consortium labs to aggressively trace, track and genetically characterize the variants and determine the case fatality rate (CFR). However, the integrated surveillance-response systems should not only be limited to wastewater, food or fomites but must also include wildlife or domestic animals as potential reservoirs of zoonotics, to prevent the outbreak at the very first place.

The way forward to curb future pandemics is "One Health Approach" integrating the well-being of the environment and biodiversity in totality. The pandemic has already warned that interfering with planetary health can result in emergence of novel diseases. Steps to safeguard the environment is the right investment to protect from future pandemics. Lately UNEP has put forward a new category of international crime- 'ecocide' (crimes against nature), elaborating our responsibilities towards nature and to stop the overexploitation. Dobson et al. (2020) predict that preventing future zoonotic outbreaks like COVID-19 through reduced deforestation and restricted wildlife trade would be just $2 \%$ of the economic and mortality costs inflicted due to COVID19 pandemic. Realizing the targets of sustainability of ecosystems and environment as whole should be the prime focus to avert the future pandemics and curb the arrival of novel zoonotic microorganisms in human population. 
There are different opinions on the origin and emergence of COVID-19, yet its rapid spread across the globe, followed by lockdowns and social restrictions have highlighted the role of environment in this pandemic. While role of anthropogenic activities and their impact on environment resulting in emergence and spread of zoonotic diseases such as COVID-19 is being studied vigorously now, positive effects of social restrictions imposed due to COVID-19 on environment have also invited huge attention among scientists and environmentalists. Almost whole planet was like a natural laboratory for studying the recovery of ecosystems and reduction of environmental pollution during the restrictions. The research in such unique conditions showed the way to achieve the targets of SDGs in the coming time. The special issue "COVID-19 and Environmental Linkages" was envisaged with the objectives to attend the issues of environmental linkages of COVID-19, addressing the global response to the pandemic, and the environmental perspectives that may be significant for making the policies to sustain the planet and avert the future biological disasters. Researchers from around the globe have contributed in the special issue focussing on the causes of onset of pandemic, its impact on the environment and future directions of research related to environmental sustainability for controlling disease outbreaks largely caused by zoonotic microorganisms.

Author contribution All authors contributed equally in writing the manuscript. NKA edited the final draft.

\section{References}

Ball P (2021) The lightning-fast quest for COVID vaccines-and what it means for other diseases. Nature 589:16-18. https://doi.org/10. 1038/d41586-020-03626-1

Dobson AP, Pimm SL, Hannah L, Kaufman L, Ahumada JA et al (2020) Ecology and economics for pandemic prevention. Science 369(6502):379-381

Hofmeister AM, Seckler JM, Criss GM (2021) Possible roles of permafrost melting, atmospheric transport, and solar irradiance in the development of major coronavirus and influenza pandemics. Int $\mathbf{J}$ Environ Res Public Health 18:3055

Joint World Health Organization-China Study team Report (2021) WHO-Convened Global Study of Origins of SARS-CoV-2: China Part. https://apo.org.au/sites/default/files/resource-files/2021-03/ apo-nid311637.pdf. Accessed 30 Mar 2021

Mishra J, Mishra P, Arora NK (2021) Linkages between environmental issues and zoonotic diseases: with reference to COVID-19 pandemic. Environ Sustain. https://doi.org/10.1007/ s42398-021-00165-x

Renninger N, Nastasi N, Bope A, Cochran SJ, Haines SR et al (2021) Indoor dust as a matrix for surveillance of COVID-19. mSystems 6(2):e01350-e1420. https://doi.org/10.1128/mSystems.01350-20

World Health Organization (2020) Water, sanitation, hygiene, and waste management for SARS-CoV-2, the virus that causes COVID-19. https://www.who.int/publications/i/item/water-sanit ation-hygiene-and-waste-management-for-the-covid-19-virusinterim-guidance. Accessed 29 July 2020

Yao L, Zhu W, Shi J, Xu T, Qu G et al (2021) Detection of coronavirus in environmental surveillance and risk monitoring for pandemic control. Chem Soc Rev 50:3656-3676

Publisher's Note Springer Nature remains neutral with regard to jurisdictional claims in published maps and institutional affiliations. 